\title{
An identification technique for the co- frequency mixed communication signals based on cumulants
}

\author{
Chaowei Duan(D, Yafeng Zhan* and Hao Liang
}

\begin{abstract}
Identification of the co-frequency interference is a common problem in wireless digital communication systems. The high-order statistics (HOS) feature based on cumulants is a widely adopted solution. However, prior knowledge of the timings and the symbol period is required to extract the cumulants, which is quite difficult in a blind environment. In order to solve this problem, this paper proposed a more general calculation for the cumulants based on the over-sampled data with consideration of the inter-symbol interference (ISI). The generalized theoretical value of the cumulants is deduced in this paper. Besides, the HOS feature based on the generalized cumulants for identification is found to be robust with different roll-off factor and sampling number per symbol. Computer simulations are performed to prove the validity of the proposed method.
\end{abstract}

Keywords: High-order statistics, Cumulants, Co-frequency mixed signal, Signal identification

\section{Introduction}

With the frequency spectrum becoming more and more intense in wireless communication, the receivers suffer from the intentional or unintentional co-frequency interference more and more frequently [1-3]. Specifically, paired carrier multiple access (PCMA) is a new technique adopted in satellite communication system, and the earth stations receive a mixed signal of two intentional co-frequency signals $[4,5]$. The conventional demodulation performance is severely degraded by the co-frequency interference, which leads emerging need for the receivers to identify the type of the received signal, a single signal or a mixed signal, before adopting corresponding procedure.

The problem of the mixed signal identification has been explored in current literatures [6-14]. The commonly adopted methods can be divided into two types. One is the information theoretic based method $[6,7]$; the other is feature-based method [8-13]. The former method adopts the correlation matrix of the received signal to estimate the number of the transmit signals. In [6], the classic Akaike information criterion (AIC) algorithm and minimum description length (MDL) are reviewed. However, these algorithms require complex

\footnotetext{
* Correspondence: zhanyf@tsinghua.edu.cn

Space Center, Tsinghua University, Haidian District, Beijing 100084, China
}

computation for eigen decomposition of the correlation matrix. Moreover, the information theoretic methods require the number of the receive antennas larger than the number of the transmit signals, which is not always satisfied.

On the other hand, the feature-based methods extract features from the received signal, which means a priori knowledge of the received signals is required. In $[8,9]$, the features of second-order cyclostationary and the spectral coherence function are adopted to detect the mixed signal. However, this method requires high computation complexity and performs poorly when the signals' carrier frequencies are nearly the same. In $[10,11]$, the cyclic stationary characteristics of digital communication signals are adopted to detect the number of the co-frequency signals. However, it fails when the cyclic frequencies are overlapped. In [12], a novel single-channel signals' number detection algorithm based on wavelet transform is investigated. The signals' symbol characteristics in Haar wavelet transform are extracted to achieve the blind estimation of the signals' number. However, the symbol rates of the signals are required to be different. In [13], the high-order moments of the received signal are employed to detect the number of the co-frequency signals. However, this 
method requires a priori information of the noise variance. Inspired by the tolerance of white Gaussian noise, the cumulants are adopted to distinguish OFDM signals from single carrier signals in [14]. However, the cumulants are extracted from a timing synchronization sampled sequence which removes the inter-symbol interference (ISI) from the over-sampled data of the received signal. That means the signal bandwidth and the timing information should be estimated if prior knowledge is not available. However, the accurate estimation of these parameters is quite difficult under low signal-to-noise ratio (SNR) condition [15-18].

In this paper, a more generalized theoretical value of the cumulants of the over-sampled data with consideration of ISI is deduced. Instead of only using the optimal sample per symbol for cumulant calculation, all the samples per symbol are used. A high-order statistics (HOS) feature based on cumulants is adopted to identify the received signal's type with prior knowledge of the modulation type of the two co-frequency signals. Moreover, the HOS feature based on the cumulants is found to be stable for arbitrary symbol period and roll-off factor of the transmitter pulse-shaping filter. Thus, it releases the need for timing synchronization and estimation of the symbol rate of the two co-frequency signals when this HOS feature is adopted to identify the received signal's type.

This paper is organized as follows. Section 1 introduces the research background. Section 2 presents the signal model and the definition of HOS. Section 3 derives the more generalized theoretical value of the cumulants of the over-sampled data with consideration of inter-symbol interference and introduces the HOS feature for signal identification. Section 4 introduces the proposed identification algorithm based on the HOS feature, and the algorithm's performance simulations are also presented. The summation is concluded in the last section.

\section{Signal model and the HOS \\ 2.1 Signal model}

In this paper, the received signal is supposed to be composed of two co-frequency signals with the same modulation type; its model can be expressed as

$$
r(t)=h_{1} e^{j\left(\omega_{1} t+\theta_{1}\right)} x_{1}(t)+h_{2} e^{j\left(\omega_{2} t+\theta_{2}\right)} x_{2}(t)+v(t)
$$

where $h_{1}$ and $h_{2}$ are the amplitude of the two signals, and the power ratio of these two signals is $P_{r}=h_{1}^{2} / h_{2}^{2}$ (for simplicity, $P_{r}$ is assumed to be larger than 1); $\omega_{1}$ and $\omega_{2}$ are the carrier frequency of the two signals and $\left|\omega_{1}-\omega_{2}\right| \ll\left|\pi / T_{1}+\pi / T_{2}\right| ; \quad T_{1}$ and $T_{2}$ are the symbol period of the two signals, respectively; $\theta_{1}$ and $\theta_{2}$ are the initial phase and are supposed to be uniform random variables on $[0,2 \pi] ; v(t)$ is an additive white Gaussian noise; $x_{1}(t)$ and $x_{2}(t)$ are the baseband modulation signals which can be described as

$$
x_{i}(t)=a_{i}(t) * g_{i}(t), i=1,2
$$

where $a_{i}(t)$ represents the modulated symbol of the $i$ th transmitted signal at time $t$ and is uniform distributed on the constellation of the modulation type; $g_{i}(t)$ represents the pulse response of the equivalent filter including forming filter, channel filter, and matched filter and is usually substituted by the finite transmitter pulse-shaping filter lasts from $-L_{1} T_{i}$ to $L_{2} T_{i}$ [19]; * represents convolution operation.

Assuming the receiver is sampled at the sampling rate $1 / T_{s}$, the over-sampled data can be written as

$$
r_{k}=h_{1} e^{j\left(\omega_{1} k T_{s}+\theta_{1}\right)} x_{1, k}+h_{2} e^{j\left(\omega_{2} k T_{s}+\theta_{2}\right)} x_{2, k}+v_{k}
$$

where $r_{k}, x_{i, k}$, and $v_{k}$ are the sampling value of $r\left(k T_{s}\right)$, $x_{i}\left(k T_{s}\right)$, and $v\left(k T_{s}\right)$, respectively.

\subsection{High-order statistics}

The HOS of $r_{k}$ can be described by the moments and the cumulants, which characterize the shape of the distribution of the signal constellation. The moments $M_{p q}$ can be obtained by calculating the expectation of $\left\{\left(r_{k}\right)^{p-q}\left(r_{k}^{*}\right)^{q}\right\}[20,21]$

$$
M_{p q}=E\left\{\left(r_{k}\right)^{p-q}\left(r_{k}^{*}\right)^{q}\right\}
$$

where (.)" represents taking the conjugate; $E\{\cdot\}$ represents the expectation operation (in the following derivation, the expectation is replaced by ergodic average).

The cumulants can be obtained by the moments using the following formulas $[20,21]$

$$
\begin{aligned}
C_{20}= & M_{20}=E\left\{\left(r_{k}\right)^{2}\right\} \\
C_{21}= & M_{21}=E\left\{r_{k} r_{k}^{*}\right\} \\
C_{42}= & M_{42}-\left|M_{20}\right|^{2}-2 M_{21}^{2} \\
= & E\left\{\left(r_{k} r_{k}^{*}\right)^{2}\right\}-E^{2}\left\{\left(r_{k}\right)^{2}\right\}-2 E^{2}\left\{r_{k} r_{k}^{*}\right\} \\
C_{63}= & M_{63}-9 C_{42} C_{21}-6 C_{21}^{3} \\
= & E\left\{\left(r_{k} r_{k}^{*}\right)^{3}\right\}-9 E\left\{\left(r_{k} r_{k}^{*}\right)^{2}\right\} E\left\{r_{k} r_{k}^{*}\right\} \\
& +9 E^{2}\left\{\left(r_{k}\right)^{2}\right\} E\left\{r_{k} r_{k}^{*}\right\}+12 E^{3}\left\{r_{k} r_{k}^{*}\right\}
\end{aligned}
$$

\section{Theoretical value of the cumulants and the HOS feature}

The studies in [20-24] have derived the theoretical value of the cumulants of the phase-shift keying (PSK) signal and the quadrature amplitude modulation (QAM) signal. However, the derivation is based on the sequence where the timing synchronization is completed and ISI is removed. 
This is not easy in practice. In this paper, we derive a more generalized theoretical value of the cumulants of the over-sampled data with ISI.

For simplicity, the additive noise $v_{k}$ is omitted during the derivation of the theoretical value. The moments $M_{p q}$ of the over-sampled data of the received signal $r(t)$ can be obtained as follows. The detail deduction is shown in Appendix 1. The deduction of the special case that the two signals are BPSK modulated and $\omega_{1}=\omega_{2}$ is given in Appendix 2.

$$
\begin{aligned}
M_{20}= & 0 \\
M_{21}= & h_{1}^{2} E\left\{x_{1, k} x_{1, k}^{*}\right\}+h_{2}^{2} E\left\{x_{2, k} x_{2, k}^{*}\right\} \\
M_{42}= & \sum_{i=1}^{2} h_{i}^{4} E\left\{\left(x_{i, k} x_{i, k}^{*}\right)^{2}\right\} \\
& +4\left(h_{1}^{2} h_{2}^{2}\right) E\left\{x_{1, k} x_{1, k}^{*}\right\} E\left\{x_{2, k} x_{2, k}^{*}\right\} \\
M_{63}= & h_{1}^{6} E\left\{\left(x_{1, k} x_{1, k}^{*}\right)^{3}\right\}+h_{2}^{6} E\left\{\left(x_{2, k} x_{2, k}^{*}\right)^{3}\right\} \\
& +9 h_{1}^{4} h_{2}^{2} E\left\{\left(x_{1, k} x_{1, k}^{*}\right)^{2}\right\} E\left\{x_{2, k} x_{2, k}^{*}\right\} \\
& +9 h_{1}^{2} h_{2}^{4} E\left\{x_{1, k} x_{1, k}^{*}\right\} E\left\{\left(x_{2, k} x_{2, k}^{*}\right)^{2}\right\}
\end{aligned}
$$

Substitute (6) into (5), the theoretical value of the cumulants $C_{p q}$ can be obtained.

$$
\begin{aligned}
C_{20}= & 0 \\
C_{21}= & h_{1}^{2} E\left\{x_{1, k} x_{1, k}^{*}\right\}+h_{2}^{2} E\left\{x_{2, k} x_{2, k}^{*}\right\} \\
C_{42}= & \sum_{i=1}^{2} h_{i}^{4} E\left\{\left(x_{i, k} x_{i, k}^{*}\right)^{2}\right\}-2 h_{i}^{4} E^{2}\left\{x_{i, k} x_{i, k}^{*}\right\} \\
C_{63}= & \sum_{i=1}^{2} h_{i}^{6} E\left\{\left(x_{i, k} x_{i, k}^{*}\right)^{3}\right\}-9 h_{i}^{6} E\left\{\left(x_{i, k} x_{i, k}^{*}\right)^{2}\right\} E\left\{\left(x_{i, k} x_{i, k}^{*}\right)\right\} \\
& +12 h_{i}^{6} E^{3}\left\{\left(x_{i, k} x_{i, k}^{*}\right)\right\}
\end{aligned}
$$

where the expectation of $\left\{\left(x_{i, k} x_{i, k}^{*}\right)^{q}\right\}$ can be referred to Appendix 1.

Seen from [21], in order to remove the influence of the signal power, a HOS feature $F_{1}$ constructed by the cumulants of the received signal is introduced for signal identification, which can be regarded as a statistics feature characterizes the shape of the distribution of the mixed signal constellation with normalized signal power.

$$
\begin{aligned}
F_{1} & =\left|\frac{C_{63}^{2}}{C_{42}^{3}}\right| \\
& =\left|\frac{\left[P_{r}^{3} E_{1,3}-9 P_{r}^{3} E_{1,2} E_{1,1}+12 P_{r}^{3} E_{1,1}^{3}+E_{2,3}-9 E_{2,2} E_{2,1}+12 E_{2,1}^{3}\right]^{2}}{\left[P_{r}^{2} E_{1,2}-2 P_{r}^{2} E_{1,1}^{2}+E_{2,2}-2 E_{2,1}^{2}\right]^{3}}\right|
\end{aligned}
$$

where $E_{i, q}=E\left\{\left(x_{i, k} x_{i, k}^{*}\right)^{q}\right\}$. The HOS feature $F_{1}$ for a single signal, i.e., $P_{r}$ approaching infinity can be obtained as follows.

$$
F_{1}=\left|\frac{\left[E_{1,3}-9 E_{1,2} E_{1,1}+12 E_{1,1}^{3}\right]^{2}}{\left[E_{1,2}-2 E_{1,1}^{2}\right]^{3}}\right|
$$

In order to verify the above theoretical derivation, Monte-Carlo method is adopted to obtain the simulation value of $F_{1}$. During the simulation, the pulse-shaping filter $g_{i}(t)$ is set to be square root raised cosine filter with $L_{1}, L_{2}$ to be 2 (for the values of $g_{i}(t)$ are quite small when $|t|$ is larger than $2 T_{i}$, so we can neglect them). Figure 1 shows the theoretical value and simulation value of $F_{1}$ of the mixed signal $r(t)$ of two co-frequency signals with the same modulation type, symbol period, and roll-off factor. The theoretical values are obtained by the following process: first, calculate the expectations of $\left\{\left(x_{i, k} x_{i, k}^{*}\right)^{q}\right\}$, using (12), (13), or (14), and then substitute them into (8) to obtain $F_{1}$. The simulation values are obtained by calculating the moments and cumulants of a generated mixed signal with different power ratio using (4) and (5), and then substitute the cumulants into (8). Seen from Fig. 1, $F_{1}$ increases with the increasing power ratio $P_{r}$. Moreover, it can be seen that the value of $F_{1}$ when $P_{r} \rightarrow \infty$

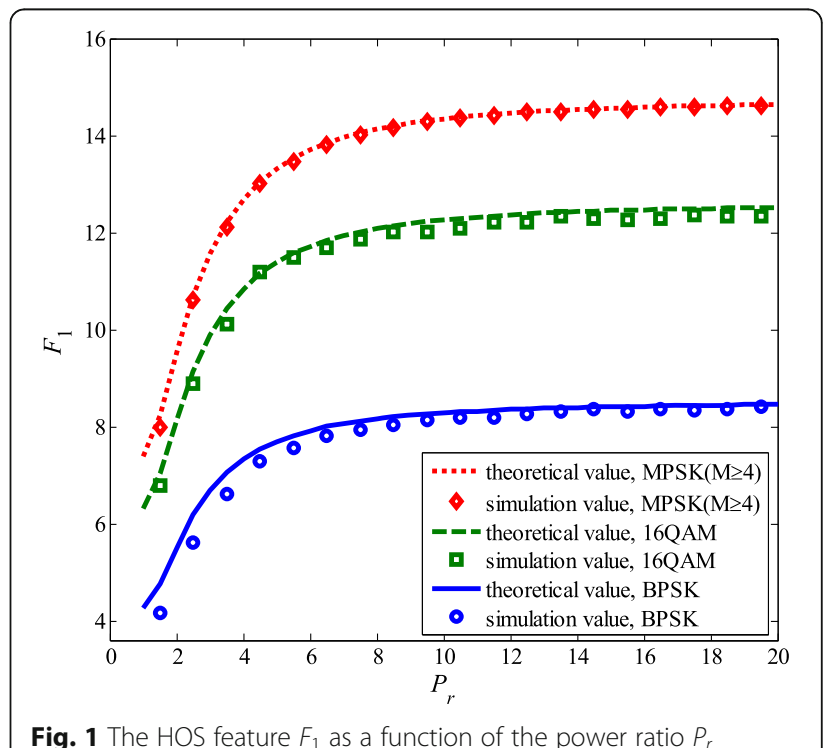


(that means the type of the received signal is a single signal) is nearly twice the value when $P_{r}=1$ (in this case, the separation of the two co-frequency signals is quite difficult). Owing to the distinctive difference of $F_{1}$ between single signal and mixed signal, $F_{1}$ can be adopted to identify the type of received signals.

Moreover, Monte-Carlo method is adopted to show the relationship between the value of $F_{1}$ and the roll-off factor $\alpha_{i}$ and the symbol period $T_{i}$ (which can be equivalent to the sampling number per symbol $N_{i}$ ). The maximum and minimum value of $F_{1}$ under different $\alpha_{i}$ and $N_{i}$ are obtained. The roll-off factor $\alpha_{i}$ is varied among $0 \sim 1$ and the sampling number per symbol $N_{i}$ is varied among 10 100. It is shown in Table 1 that the difference between the maximum and minimum value of the HOS feature $F_{1}$ is quite small, which means the roll-off factor $\alpha_{i}$ and the symbol period $T_{i}$ have little impact on the value of $F_{1}$. That releases the demand for estimation of the two signals' bandwidths, the roll-off factors, and the timing information when adopting $F_{1}$ to identify the received signal's type. Thus, it makes the HOS feature $F_{1}$ more robust in a blind environment (the special case that the two signals are BPSK modulated and $\omega_{1}=\omega_{2}$ is not taken into consideration).

\section{Results}

\subsection{A more generalized identification method based on the HOS feature}

In this section, a variety of simulation experiments are presented to illustrate the performance of the proposed identification method. The total block diagram of the identification method is shown in Fig. 2. First, the HOS feature $F_{1}$ is extracted from the over-sampled data of received signal $r(t)$ and then compared with a threshold $\eta$. If $F_{1} \geq \eta$, the type of the received signal is identified as a single signal; else if $F_{1}<\eta$, the type of the received signal is identified as a mixed signal. For the value of $F_{1}$ only

Table 1 The HOS feature $F_{1}$ under different $a_{i}$ and $N_{i}$

\begin{tabular}{lllll}
\hline Modulation type & $\begin{array}{l}\text { Roll-off } \\
\text { Factor } a_{i}\end{array}$ & $\begin{array}{l}\text { Sampling number } \\
\text { per symbol } N_{i}\end{array}$ & $\begin{array}{l}\text { Maximum } \\
\text { value of } F_{1}\end{array}$ & $\begin{array}{l}\text { Minimum } \\
\text { value of } F_{1}\end{array}$ \\
\hline BPSK, $\operatorname{Pr}=20$ & $0 \sim 1$ & $10 \sim 100$ & 8.9145 & 7.6102 \\
BPSK, $\operatorname{Pr}=5$ & $0 \sim 1$ & $10 \sim 100$ & 8.4591 & 7.0491 \\
BPSK, $\operatorname{Pr}=1$ & $0 \sim 1$ & $10 \sim 100$ & 4.6905 & 3.9025 \\
MPSK $(M \geq 4)$, & $0 \sim 1$ & $10 \sim 100$ & 14.7272 & 13.9162 \\
$\operatorname{Pr}=20$ & & & & \\
MPSK$(M \geq 4)$, & $0 \sim 1$ & $10 \sim 100$ & 14.1956 & 12.0912 \\
$\operatorname{Pr}=5$ & & & & \\
MPSK(M $\geq 4)$, & $0 \sim 1$ & $10 \sim 100$ & 8.2207 & 7.3646 \\
$\operatorname{Pr}=1$ & & & & \\
16QAM, $\operatorname{Pr}=20$ & $0 \sim 1$ & $10 \sim 100$ & 12.6301 & 11.8496 \\
16QAM, $\operatorname{Pr}=5$ & $0 \sim 1$ & $10 \sim 100$ & 12.1749 & 10.2950 \\
16QAM, $\operatorname{Pr}=1$ & $0 \sim 1$ & $10 \sim 100$ & 7.0415 & 6.2780 \\
\hline
\end{tabular}

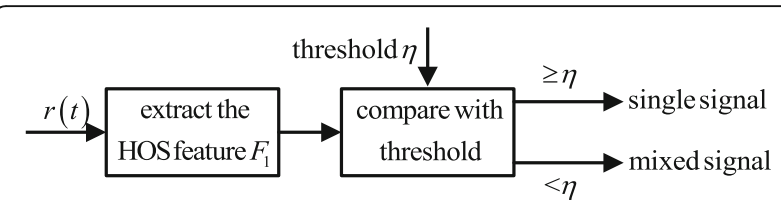

Fig. 2 The block diagram of the proposed identification method

vary with the power ratio $P_{n}$, the threshold's value can be substituted by the theoretical value of $F_{1}$ with the given modulation type of the two co-frequency signals and the power ratio $P_{r 0}$. The mixed signal can be regarded as a single signal when the two signals' power ratio $P_{r}$ is larger than $P_{r 0}$ (in this paper, $P_{r 0}$ is set as 10 for the bit-error rate (BER) performance degradation in the presence of a co-frequency interference is quite small when the power ratio $P_{r}$ is larger than 10 [25]). This proposed identification method is also available for the cases that the mixed signal is composed of more than two co-frequency signals.

The SNR of the mixed signal is defined as.

$$
\mathrm{SNR}=E_{s 1} / N_{0}
$$

where $E_{s i}$ is the energy per symbol of the $i$ th signal. $N_{0}$ is the noise power spectral density.

In experiment 1 , we consider the identification performance under different SNR condition. To evaluate the performance, the probability of correct identification $P_{C I}$ is counted by 500 times Monte-Carlo simulations. The simulation parameters are set as follows: the carrier frequencies $\left(\omega_{1} / 2 \pi, \omega_{2} / 2 \pi\right)$ are set as $(100 \mathrm{e} 6+8 \mathrm{e} 3 \mathrm{~Hz}$, $100 \mathrm{e} 6-6 \mathrm{e} 3 \mathrm{~Hz})$; the baud rates $\left(1 / T_{1}, 1 / T_{2}\right)$ are set as (10e6 bps, 10e6 bps) and (10e6 bps, 7e6 bps) for the two signals with same symbol period case and with different symbol period case, respectively; the sampling rate is set as $1400 \mathrm{e} 6 \mathrm{~Hz}$; the total symbol number used for simulation is set as 5e4. Figure 3 shows the identification performance when the received signal is a mixed signal with the power ratio $P_{r}$ to be 5 , and Fig. 4 shows the identification performance when the received signal is a single signal. Obviously, the $P_{C I}$ increases with the increasing SNR and the identification method is robust for the two signals with different symbol period case. Seen from these figures, it is obvious that the proposed identification method performs better under a higher SNR condition.

In experiment 2 , we consider the identification performance under different power ratio $P_{r}$ of the two signals. The simulation parameters are set as experiment 1 . The identification performance for the $\mathrm{SNR}=0 \mathrm{~dB}$ is shown in Fig. 5. It is shown that the identification performance decreases with the increasing $P_{r}$ for the value of the extracted HOS feature $F_{1}$ gets closer to the threshold $\eta$. 


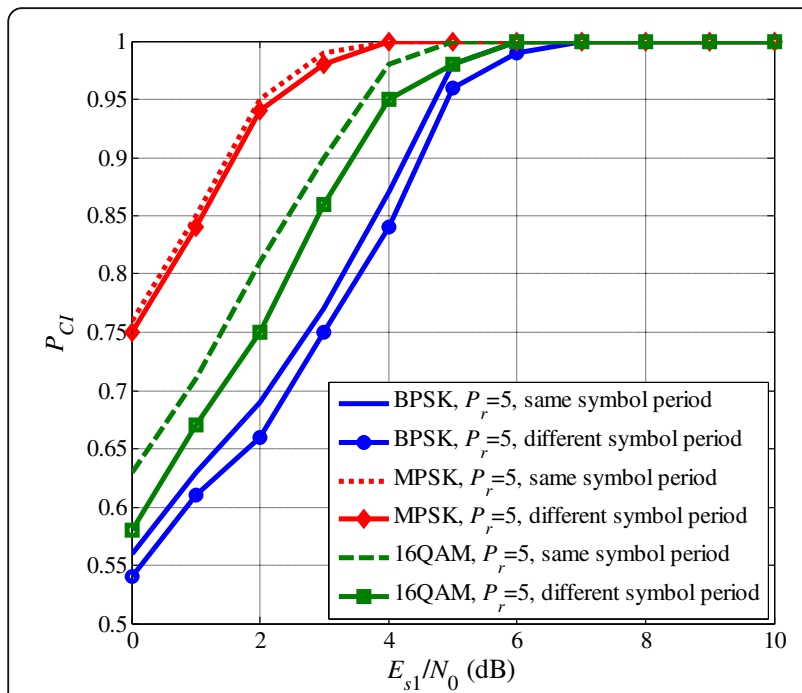

Fig. 3 The $P_{C l}$ as a function of the SNR for the identification of a mixed signal

In experiment 3, we consider the identification performance under different total symbol number used for simulation. Figure 6 shows the identification performance for the $\mathrm{SNR}=6 \mathrm{~dB}$ and the received signal is a single signal; it is clear to see that the identification performance increases with the increasing total symbol number used for simulation.

In experiment 4, the identification performance comparison between the proposed HOS feature-based method and the cyclostationary spectrum coherence function (SOF)-based method [8, 9] is made. For the two co-frequency, signals' carrier frequencies are set to be too close

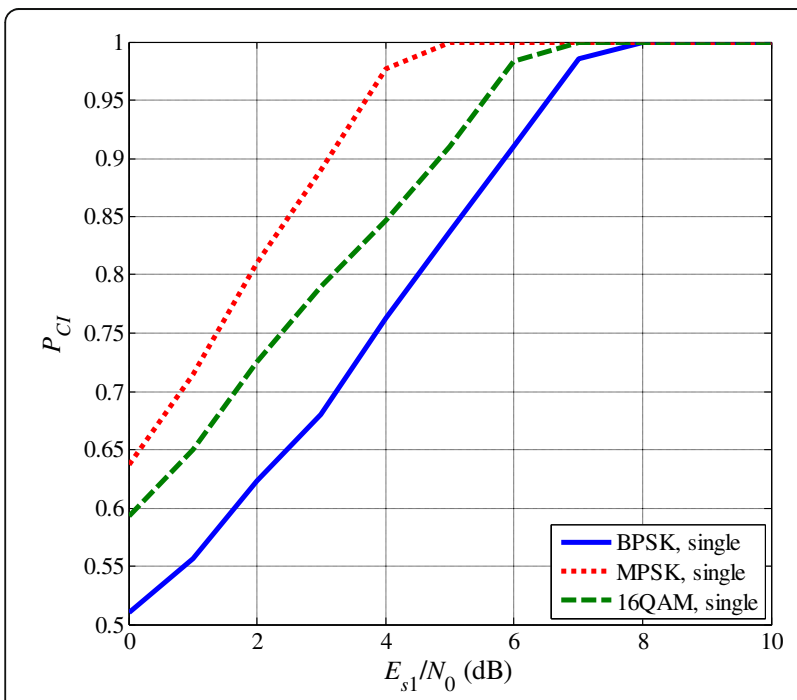

Fig. 4 The $P_{C l}$ as a function of the SNR for the identification of a single signal

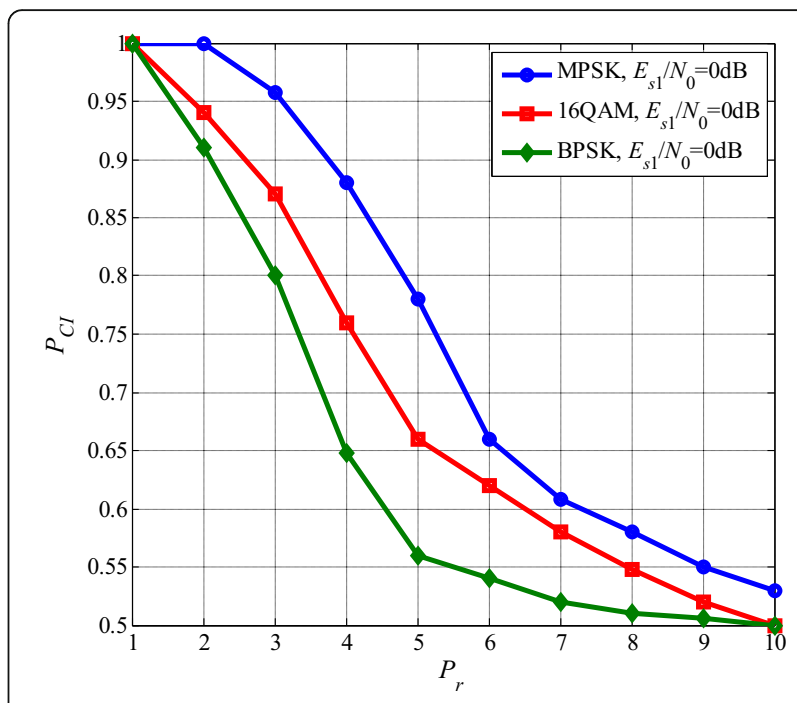

Fig. 5 The $P_{C l}$ as a function of the power ratio $P_{r}$

in experiment 1 experiment 3 and the SOF-based method fails under that condition, the carrier frequencies $\left(\omega_{1} / 2 \pi, \omega_{2} / 2 \pi\right)$ are reset as $(100 \mathrm{e} 6+8 \mathrm{e} 3 \mathrm{~Hz}$, $102 \mathrm{e} 6-6 \mathrm{e} 3 \mathrm{~Hz}$ ) in this experiment. Moreover, for the SOF-based method that requires prior knowledge of the carrier frequencies, symbol period, we suppose those knowledge are known during our simulation for simplicity. The result is shown in Fig. 7. Seen from Fig. 7, it is obvious that our proposed method performs much better than the SOF-based method under low SNR condition and our proposed method is more robust in a blind environment.

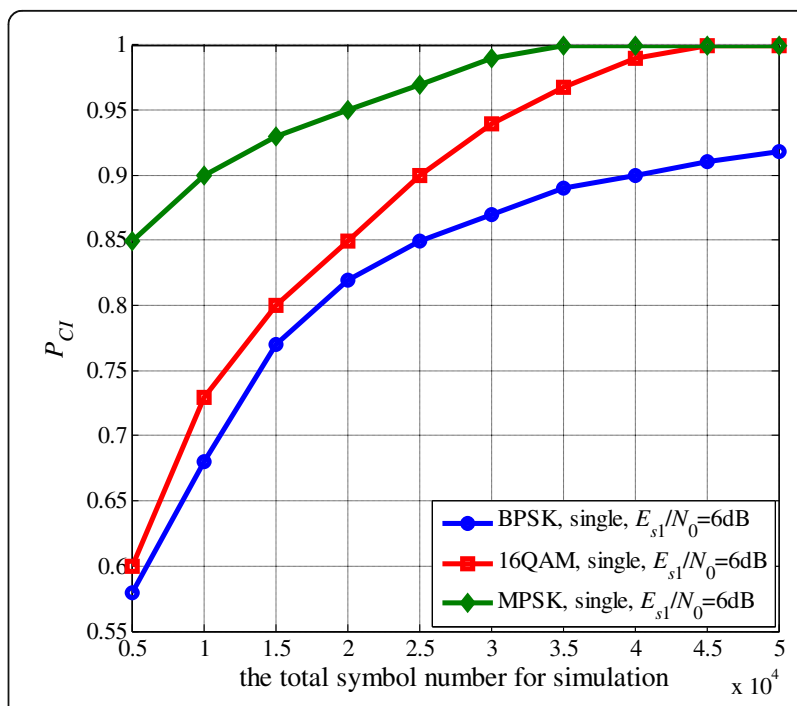

Fig. 6 The $P_{C l}$ as a function of the total symbol number for simulation 


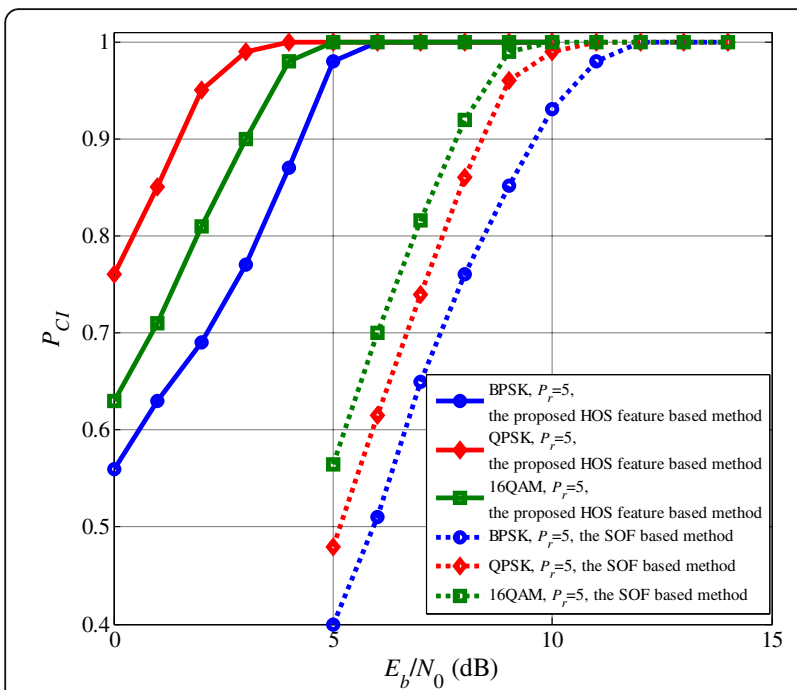

Fig. 7 The performance comparison between the proposed HOS feature-based method and the SOF-based method

\section{Discussion}

This paper derives the theoretical value of the cumulants of the whole sampled data with consideration of the inter-symbol interference. The HOS feature is adopted to identify the co-frequency mixed signal. Since it releases the need for timing synchronization of the received signal, it makes the HOS feature more fitful in blind environment. However, this proposed method requires prior knowledge of the modulation type of the two co-frequency signals.

\section{Conclusions}

In this paper, a more generalized theoretical value of the cumulants and a HOS feature are derived based on the over-sampled data. It is effective for the release of the demand for prior knowledge of timing information and symbol periods in current literatures [14, 20-24]. Based on the derived HOS feature, a novel identification technique for the co-frequency mixed signals with the same modulation type is proposed. This approach is robust for arbitrary cases of the co-frequency signals with different roll-off factor, sampling number per symbol, and power ratio.

Furthermore, the derived cumulants in this paper can be applied in blind modulation classification of a single signal for the needless of prior knowledge and estimation of the power ratio of the mixed signal for the power control in cellular systems [26-28]. Further discussions could be made in the future.

\section{Methods}

This paper studies the general theoretical value of the cumulants of the over-sampled received data and adopts the HOS feature to identify the co-frequency mixed signal. The proposed identification method's performance is obtained by Monte-Carlo simulations using MATLAB software.

\section{Appendix 1}

For simplification, the additive noise $v_{k}$ is omitted during the derivation of the theoretical value of the moments. Seen from (3), the product of the over-sampled data $r_{k}$ and its conjugation can be written as follows.

$$
\begin{aligned}
r_{k} r_{k}^{*}= & h_{1}^{2} x_{1, k} x_{1, k}^{*}+h_{2}^{2} x_{2, k} x_{2, k}^{2} \\
& +h_{1} h_{2} e^{j\left[\left(\omega_{1}-\omega_{2}\right) k T_{s}+\left(\theta_{1}-\theta_{2}\right)\right]} x_{1, k} x_{2, k}^{*} \\
& +h_{1} h_{2} e^{-j\left[\left(\omega_{1}-\omega_{2}\right) k T_{s}+\left(\theta_{1}-\theta_{2}\right)\right]} x_{1, k}^{*} x_{2, k}
\end{aligned}
$$

Assume $k=N_{i}(m-1)+p$ (where $N_{i}$ is the sampling number per symbol of the $i$ th transmitted signal; $m$ is the $m$ th transmitted symbol of the $i$ th transmitted signal at time $k T_{s}$ ). Then, the baseband modulation signal $x_{i, k}$ can be extended as follows.

$$
\begin{aligned}
x_{i, k}= & a_{i}(k) * g_{i}(k) \\
= & \sum_{n=1}^{\left(L_{1}+L_{2}\right) N_{i}+1} g_{i}(n) a_{i}\left(N_{i}(m-1)+p-n+1+\left(L_{1}+L_{2}\right) N_{i} / 2\right) \\
= & {\left[g_{i}\left(N_{i}\left(L_{2}+L_{1}-1\right)+1+p\right)+\cdots\right.} \\
& \left.+g_{i}\left(N_{i}\left(L_{2}+L_{1}\right)+1+p\right)\right] a_{i, m-L_{1}}+\left[g _ { i } \left(N_{i}\left(L_{2}+L_{1}-2\right)\right.\right. \\
& \left.+1+p)+\cdots+g_{i}\left(N_{i}\left(L_{2}+L_{1}-1\right)+p\right)\right] a_{i, m-L_{1}+1}+\cdots \\
& +\left[g_{i}\left(N_{i}\left(L_{2}-1\right)+1+p\right)+\cdots+g_{i}\left(N_{i} L_{2}+p\right)\right] a_{i, m}+\cdots \\
& +\left[g_{i}(1)+\cdots+g_{i}(p)\right] a_{i, m+L_{2}}
\end{aligned}
$$

where $a_{i}(k)$ and $g_{i}(k)$ is assumed to be $a_{i}\left(k T_{s}\right)$ and $g_{i}\left((k-1) T_{s}-L_{1} T_{i}\right)$, respectively; $a_{i, m}$ is the $m$ th transmitted symbol of the $i$ th modulated baseband signal; the finite pulse-shaping filter $g_{i}(k)$ lasts from $-L_{1} T_{i}$ to $L_{2} T_{i}$.

In the case of MPSK-modulated baseband signal, the expectation of $\left\{\left(x_{i, k} x_{i, k}^{*}\right)^{q}\right\}(q \leq 3)$ can be written as (13), where $q_{1}, \cdots, q_{L_{1}+L_{2}+1}$ are positive integers; the expectation of \{ $\left.a_{i, m} a_{i, m}^{*}\right\}$ is assumed to be 1 ; $C_{q}^{q_{1}}$ denotes binomial coefficient. Note that when $a_{i, m}$ is BPSK modulated, $a_{i, m}^{*}=a_{i, m}$. Then, the expectation of $\left\{\left(x_{i, k} x_{i, k}^{*}\right)^{q}\right\}$ of BPSK-modulated signal is different from (13), which is deduced in (14) (where $q_{1}, \cdots, q_{L_{1}+L_{2}+1}$ are positive even integers).

While in the case of QAM-modulated baseband signal, $a_{i, m}=I_{i, m}+j Q_{i, m}$ (where $I_{i, m}$ and $Q_{i, m}$ are independent with each other and take $\pm 1, \pm 3$, etc. with equal probability). Then, the expectation of $\left\{\left(x_{i, k} x_{i, k}^{*}\right)^{q}\right\}(q \leq 3)$ should be written in another form. 


$$
\begin{aligned}
E\left\{\left(x_{i, k} x_{i, k}^{*}\right)^{q}\right\}= & \sum_{p=1}^{N_{i}} \sum_{\left\{q_{1}+\cdots+q_{L_{1}+L_{2}+1}=q\right\}} \\
& \left\{C _ { q } ^ { q _ { 1 } \cdots C _ { q - q _ { 1 } \cdots \cdots } ^ { q _ { L _ { 1 } + L _ { 2 } + 1 } } - q _ { L _ { 1 } + L _ { 2 } } } \left[g _ { i } \left(N_{i}\left(L_{2}+L_{1}-1\right)\right.\right.\right. \\
& \left.+1+p)+\cdots+g_{i}\left(N_{i}\left(L_{2}+L_{1}\right)+1+p\right)\right]^{q_{1}} \\
& \times\left[g_{i}\left(N_{i}\left(L_{2}+L_{1}-2\right)+1+p\right)+\cdots\right. \\
& \left.+g_{i}\left(N_{i}\left(L_{2}+L_{1}-1\right)+p\right)\right]^{q_{2}} \times \cdots \\
& \times\left[g_{i}\left(N_{i}\left(L_{2}-1\right)+1+p\right)+\cdots\right. \\
& \left.+g_{i}\left(N_{i} L_{2}+p\right)\right]^{q_{L_{1}+1}} \times \cdots \times\left[g_{i}(1)+\cdots\right. \\
& \left.\left.+g_{i}(p)\right]^{q_{L_{1}+L_{2}+1}}\right\}^{2} / N_{i}
\end{aligned}
$$

$$
\begin{aligned}
E\left\{\left(x_{i, k} x_{i, k}^{*}\right)^{q}\right\}= & E\left\{\left(x_{i, k}\right)^{2 q}\right\} \\
= & \sum_{p=1}^{N_{i}} \sum_{\left\{q_{1}+\cdots+q_{L_{1}+L_{2}+1}=2 q\right\}} C_{2 q}^{q_{1} \cdots} C_{2 q-\cdots-q_{L_{1}+L_{2}}}^{q_{L_{1}}+L_{2}+1} \\
& {\left[g_{i}\left(N_{i}\left(L_{2}+L_{1}-1\right)+1+p\right)+\cdots\right.} \\
& \left.+g_{i}\left(N_{i}\left(L_{2}+L_{1}\right)+p\right)\right]^{q_{1}} \times \cdots \\
& \times\left[g_{i}\left(N_{i}\left(L_{2}-1\right)+1+p\right)+\cdots+g_{i}\left(N_{i} L_{2}+p\right)\right]^{q_{L_{1}+1}} \\
& \times \cdots \times\left[g_{i}(1)+\cdots+g_{i}(p)\right]^{q_{L_{1}+L_{2}+1}} / N_{i}
\end{aligned}
$$$$
E\left\{\left(x_{i, k} x_{i, k}^{*}\right)^{q}\right\}=E\left\{\left[\left(I_{i}(k) * h_{i}(k)\right)^{2}+\left(Q_{i}(k) * h_{i}(k)\right)^{2}\right]^{q}\right\}
$$

$$
\begin{aligned}
E\{( & \left.\left(I_{i}(k) * g_{i}(k)\right)^{2 q}\right\}=E\left\{\left(Q_{i}(k) * g_{i}(k)\right)^{2 q}\right\} \\
= & \sum_{p=1}^{N_{i}} \sum_{\left\{q_{1}+\cdots+q_{L_{1}+L_{2}+1}=2 q\right\}} C_{2 q}^{q_{1} \cdots C_{2 q-\cdots-q_{L_{1}+L_{2}}}^{q_{L_{1}+L_{2}+1}}} \\
& {\left[g_{i}\left(N_{i}\left(L_{2}+L_{1}-1\right)+1+p\right)+\cdots\right.} \\
& \left.+g_{i}\left(N_{i}\left(L_{2}+L_{1}\right)+p\right)\right]^{q_{1}} \times \cdots \\
& \times\left[g_{i}\left(N_{i}\left(L_{2}-1\right)+1+p\right)+\cdots\right. \\
& \left.+g_{i}\left(N_{i} L_{2}+p\right)\right]^{q_{L_{1}+1}} \times \cdots \\
& \times\left[g_{i}(1)+\cdots+g_{i}(p)\right]^{q_{L_{1}+L_{2}+1}} \times E\left[I_{i, m}^{q_{1}}\right] \times \cdots \\
& \times E\left[I_{i, m}^{q_{L_{1}+L_{2}+1}}\right] / N_{i}
\end{aligned}
$$

where the expectation of $\left\{\left(I_{i}(k) * h_{i}(k)\right)^{2 q}\right\}$ and $\left\{\left(Q_{i}(k) *\right.\right.$ $\left.\left.h_{i}(k)\right)^{2 q}\right\}$ can be obtained as (16), where $q_{1}, \cdots, q_{L_{1}+L_{2}+1}$ are positive even integers.
Given (11), (13), (14), (15), and (16), the moments $M_{p q}$ of the over-sampled data can be written as follows.

$$
\begin{aligned}
M_{20}= & E\left\{h_{1}^{2} e^{j 2\left(\omega_{1} k T_{s}+\theta_{1}\right)} x_{1, k}^{2}+h_{2}^{2} e^{j 2\left(\omega_{2} k T_{s}+\theta_{2}\right)} x_{2, k}^{2}\right. \\
& \left.+2 h_{1} h_{2} e^{\left.j\left(\omega_{1}+\omega_{2}\right) k T_{s}+\theta_{1}+\theta_{2}\right]} x_{1, k} x_{2, k}\right\}=0 \\
M_{21}= & h_{1}^{2} E\left\{x_{1, k} x_{1, k}^{*}\right\}+h_{2}^{2} E\left\{x_{2, k} x_{2, k}^{*}\right\} \\
M_{42}= & h_{1}^{4} E\left\{\left(x_{1, k} x_{1, k}^{*}\right)^{2}\right\}+h_{2}^{4} E\left\{\left(x_{2, k} x_{2, k}^{*}\right)^{2}\right\} \\
& +4\left(h_{1}^{2} h_{2}^{2}\right) E\left\{x_{1, k} x_{1, k}^{*}\right\} E\left\{x_{2, k} x_{2, k}^{*}\right\} \\
& +2\left(h_{1}^{2} h_{2}^{2}\right) E\left\{\operatorname{Re}\left[e^{j\left[2\left(\omega_{1}-\omega_{2}\right) k T_{s}+2\left(\theta_{1}-\theta_{2}\right)\right]} x_{1, k}^{2}\left(x_{2, k}^{*}\right)^{2}\right]\right\} \\
M_{63}= & h_{1}^{6} E\left\{\left(x_{1, k} x_{1, k}^{*}\right)^{3}\right\}+h_{2}^{6} E\left\{\left(x_{2, k} x_{2, k}^{*}\right)^{3}\right\} \\
& +9 h_{1}^{4} h_{2}^{2} E\left\{\left(x_{1, k} x_{1, k}^{*}\right)^{2}\right\} E\left\{x_{2, k} x_{2, k}^{*}\right\} \\
& +9 h_{1}^{2} h_{2}^{4} E\left\{x_{1, k} x_{1, k}^{*}\right\} E\left\{\left(x_{2, k} x_{2, k}^{*}\right)^{2}\right\} \\
& +6 h_{1}^{4} h_{2}^{2} E\left\{\operatorname{Re}\left[e^{j\left[2\left(\omega_{1}-\omega_{2}\right) k T_{s}+2\left(\theta_{1}-\theta_{2}\right)\right]} x_{1, k}^{3} x_{1, k}^{*}\left(x_{2, k}^{*}\right)^{2}\right]\right\} \\
& +6 h_{1}^{2} h_{2}^{4} E\left\{\operatorname{Re}\left[e^{j\left[2\left(\omega_{1}-\omega_{2}\right) k T_{s}+2\left(\theta_{1}-\theta_{2}\right)\right]} x_{1, k}^{2} x_{2, k}\left(x_{2, k}^{*}\right)^{3}\right]\right\}
\end{aligned}
$$

Note that the expectation of the term $E\{\operatorname{Re}[\cdot]\}$ is zero except when $\omega_{1}-\omega_{2}=0$ and the two baseband signal $x_{i, k}$ are BPSK modulated. The HOS of the over-sampled data $r_{k}$ when the two baseband signal $x_{i, k}$ are BPSK modulated and $\omega_{1}-\omega_{2}=0$ is discussed in Appendix 2 .

\section{Appendix 2}

When the two baseband signal $x_{i, k}$ are BPSK modulated, $x_{i, k}^{*}=x_{i, k}$. The expectation of the term $E\{\operatorname{Re}[\cdot]\}$ in (17) can be obtained as (18).

Because $E\left\{\cos \left(2\left(\omega_{1}-\omega_{2}\right) k T_{s}+2\left(\theta_{1}-\theta_{2}\right)\right)\right\}$ is obtained by calculating the ergodic average of $\cos \left(2\left(\omega_{1}-\omega_{2}\right) k T_{s}+\right.$ $\left.2\left(\theta_{1}-\theta_{2}\right)\right)$ as shown in (19).

It is easy to see that $E\left\{\cos \left(2\left(\omega_{1}-\omega_{2}\right) k T_{s}+2\left(\theta_{1}-\theta_{2}\right)\right)\right\}$ is not zero only when $\omega_{1}=\omega_{2}$.

$$
\begin{aligned}
E\{ & \left.\operatorname{Re}\left[e^{j\left[2\left(\omega_{1}-\omega_{2}\right) k T_{s}+2\left(\theta_{1}-\theta_{2}\right)\right]} x_{1, k}^{2}\left(x_{2, k}^{*}\right)^{2}\right]\right\} \\
= & E\left\{x_{1, k} x_{1, k}^{*}\right\} E\left\{x_{2, k} x_{2, k}^{*}\right\} E\left\{\cos \left(2\left(\omega_{1}-\omega_{2}\right) k T_{s}+2\left(\theta_{1}-\theta_{2}\right)\right)\right\} \\
E & \left\{\operatorname{Re}\left[e^{j\left[2\left(\omega_{1}-\omega_{2}\right) k T_{s}+2\left(\theta_{1}-\theta_{2}\right)\right]} x_{1, k}^{3} x_{1, k}^{*}\left(x_{2, k}^{*}\right)^{2}\right]\right\} \\
= & E\left\{\left(x_{1, k} x_{1, k}^{*}\right)^{2}\right\} E\left\{x_{1, k} x_{1, k}^{*}\right\} E\left\{\cos \left(2\left(\omega_{1}-\omega_{2}\right) k T_{s}+2\left(\theta_{1}-\theta_{2}\right)\right)\right\} \\
& E\left\{\operatorname{Re}\left[e^{j\left[2\left(\omega_{1}-\omega_{2}\right) k T_{s}+2\left(\theta_{1}-\theta_{2}\right)\right]} x_{1, k}^{2} x_{2, k}\left(x_{2, k}^{*}\right)^{3}\right]\right\}=E\left\{x_{1, k} x_{1, k}^{*}\right\} \\
& E\left\{\left(x_{2, k} x_{2, k}^{*}\right)^{2}\right\} E\left\{\cos \left(2\left(\omega_{1}-\omega_{2}\right) k T_{s}+2\left(\theta_{1}-\theta_{2}\right)\right)\right\}
\end{aligned}
$$




$$
E\left\{\cos \left(2\left(\omega_{1}-\omega_{2}\right) k T_{s}+2\left(\theta_{1}-\theta_{2}\right)\right)\right\}
$$$$
=\frac{1}{N} \sum_{k=0}^{N-1} \cos \left(2\left(\omega_{1}-\omega_{2}\right) k T_{s}+2\left(\theta_{1}-\theta_{2}\right)\right)
$$

$$
\begin{gathered}
E\left\{\cos \left(2\left(\omega_{1}-\omega_{2}\right) k T_{s}+2\left(\theta_{1}-\theta_{2}\right)\right)\right\} \\
\quad=\cos \left(2\left(\theta_{1}-\theta_{2}\right)\right), \omega_{1}=\omega_{2}
\end{gathered}
$$

$$
\begin{aligned}
M_{20}= & E\left\{h_{1}^{2} e^{j 2\left(\omega_{1} k T_{s}+\theta_{1}\right)} x_{1, k}^{2}+h_{2}^{2} e^{j 2\left(\omega_{2} k T_{s}+\theta_{2}\right)} x_{2, k}^{2}\right. \\
& \left.+2 h_{1} h_{2} e^{j\left[\left(\omega_{1}+\omega_{2}\right) k T_{s}+\theta_{1}+\theta_{2}\right]} x_{1, k} x_{2, k}\right\}=0 \\
M_{21}= & h_{1}^{2} E\left\{x_{1, k} x_{1, k}^{*}\right\}+h_{2}^{2} E\left\{x_{2, k} x_{2, k}^{*}\right\} \\
M_{42}= & h_{1}^{4} E\left\{\left(x_{1, k} x_{1, k}^{*}\right)^{2}\right\}+h_{2}^{4} E\left\{\left(x_{2, k} x_{2, k}^{*}\right)^{2}\right\} \\
& +4\left(h_{1}^{2} h_{2}^{2}\right) E\left\{x_{1, k} x_{1, k}^{*}\right\} E\left\{x_{2, k} x_{2, k}^{*}\right\} \\
& +2\left(h_{1}^{2} h_{2}^{2}\right) E\left\{x_{1, k} x_{1, k}^{*}\right\} E\left\{x_{2, k} x_{2, k}^{*}\right\} \cos \left(2\left(\theta_{1}-\theta_{2}\right)\right) \\
M_{63}= & h_{1}^{6} E\left\{\left(x_{1, k} x_{1, k}^{*}\right)^{3}\right\}+h_{2}^{6} E\left\{\left(x_{2, k} x_{2, k}^{*}\right)^{3}\right\} \\
& +9 h_{1}^{4} h_{2}^{2} E\left\{\left(x_{1, k} x_{1, k}^{*}\right)^{2}\right\} E\left\{x_{2, k} x_{2, k}^{*}\right\} \\
& +9 h_{1}^{2} h_{2}^{4} E\left\{x_{1, k} x_{1, k}^{*}\right\} E\left\{\left(x_{2, k} x_{2, k}^{*}\right)^{2}\right\} \\
& +6 h_{1}^{4} h_{2}^{2} E\left\{\left(x_{1, k} x_{1, k}^{*}\right)^{2}\right\} E\left\{x_{2, k} x_{2, k}^{*}\right\} \cos \left(2\left(\theta_{1}-\theta_{2}\right)\right) \\
& +6 h_{1}^{2} h_{2}^{4} E\left\{x_{1, k} x_{1, k}^{*}\right\} E\left\{\left(x_{2, k} x_{2, k}^{*}\right)^{2}\right\} \cos \left(2\left(\theta_{1}-\theta_{2}\right)\right) \\
&
\end{aligned}
$$

Given (17), (18), and (20), the moments $M_{p q}$ of the over-sampled data when the two signals are BPSK modulated and $\omega_{1}=\omega_{2}$ can be obtained as (21).

Substitute (21) into (5), the theoretical value of the cumulants $C_{p q}$ when the two signals are BPSK modulated and $\omega_{1}=\omega_{2}$ can be obtained as (22).

It is obvious that the value of $F_{1}$ varies with different values of $\theta_{1}-\theta_{2}$ when the two signals are BPSK modulated and $\omega_{1}=\omega_{2}$. The theoretical values and the simulation values of $F_{1}$ under different values of $\theta_{1}-\theta_{2}$ are shown in Fig. 8. It is easy to see that our proposed identification method fails because the value of $F_{1}$ is changeable. However, this condition that the two signals' carrier frequencies are exactly the same is quite hard to reach in practice. So our proposed identification method is still acceptable.

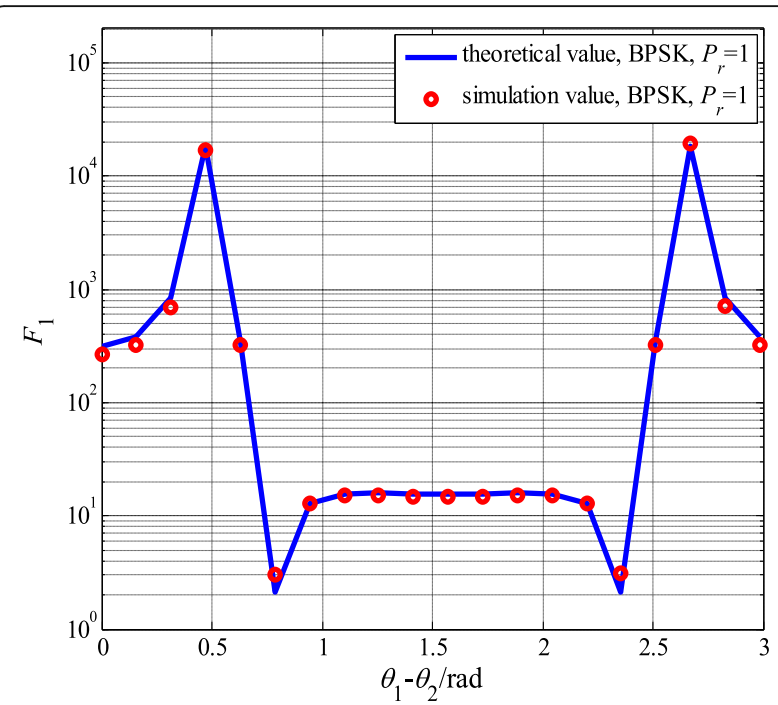

Fig. 8 The HOS feature $F_{1}$ as a function of the phase difference when the two signals are BPSK modulated and $\omega_{1}=\omega_{2}$

$$
\begin{aligned}
C_{20}= & \\
C_{21}= & h_{1}^{2} E\left\{x_{1, k} x_{1, k}^{*}\right\}+h_{2}^{2} E\left\{x_{2, k} x_{2, k}^{*}\right\} \\
C_{42}= & \sum_{i=1}^{2}\left(h_{i}^{4} E\left\{\left(x_{i, k} x_{i, k}^{*}\right)^{2}\right\}-2 h_{i}^{4} E^{2}\left\{x_{i, k} x_{i, k}^{*}\right\}\right) \\
& +2 h_{1}^{2} h_{2}^{2} E\left\{x_{1, k} x_{1, k}^{*}\right\} E\left\{x_{2, k} x_{2, k}^{*}\right\} \cos \left(2\left(\theta_{1}-\theta_{2}\right)\right) \\
C_{63}= & \sum_{i=1}^{2}\left(h_{i}^{6} E\left\{\left(x_{i, k} x_{i, k}^{*}\right)^{3}\right\}-9 h_{i}^{6} E\left\{\left(x_{i, k} x_{i, k}^{*}\right)^{2}\right\}\right. \\
& \left.E\left\{\left(x_{i, k} x_{i, k}^{*}\right)\right\}+12 h_{i}^{6} E^{3}\left\{\left(x_{i, k} x_{i, k}^{*}\right)\right\}\right) \\
& +6 h_{1}^{4} h_{2}^{2} E\left\{\left(x_{1, k} x_{1, k}^{*}\right)^{2}\right\} E\left\{x_{2, k} x_{2, k}^{*}\right\} \cos \left(2\left(\theta_{1}-\theta_{2}\right)\right) \\
& +6 h_{1}^{2} h_{2}^{4} E\left\{x_{1, k} x_{1, k}^{*}\right\} E\left\{\left(x_{2, k} x_{2, k}^{*}\right)^{2}\right\} \cos \left(2\left(\theta_{1}-\theta_{2}\right)\right) \\
& -18 h_{1}^{4} h_{2}^{2} E^{2}\left\{x_{1, k} x_{1, k}^{*}\right\} E\left\{x_{2, k} x_{2, k}^{*}\right\} \cos \left(2\left(\theta_{1}-\theta_{2}\right)\right) \\
& -18 h_{1}^{2} h_{2}^{4} E\left\{x_{1, k} x_{1, k}^{*}\right\} E^{2}\left\{x_{2, k} x_{2, k}^{*}\right\} \cos \left(2\left(\theta_{1}-\theta_{2}\right)\right)
\end{aligned}
$$

\section{Abbreviations}

AIC: Akaike information criterion; BER: Bit-error rate; HOS: High-order statistics; ISI: Inter-symbol interference; MDL: Minimum description length; PCMA: Paired carrier multiple access; PSK: Phase-shift keying; QAM: Quadrature amplitude modulation; SNR: Signal-to-noise ratio; SOF: Cyclostationary spectrum coherence function

\section{Funding}

This work was supported by the National Natural Science Foundation of China (61271265 and 61671263) and Tsinghua University Independent Scientific Research Project (20161080057).

Availability of data and materials

Please contact the author for data requests. 


\section{Authors' contributions}

CWD and YFZ proposed the original idea and designed the experiments. CWD and HL performed the experiments and analyzed the results. All authors read and approved the final manuscript.

\section{Competing interests}

The authors declare that they have no competing interests.

\section{Publisher's Note}

Springer Nature remains neutral with regard to jurisdictional claims in published maps and institutional affiliations.

Received: 8 April 2018 Accepted: 20 July 2018

Published online: 31 July 2018

\section{References}

1. M Aksoy et al., L-band radio-frequency interference observations during the SMAP validation experiment 2012. IEEE Trans. Geosci. Remote Sens. 54(3), 1323-1335 (2016)

2. F Wei et al., When mmWave communications meet network densification: a scalable interference coordination perspective. IEEE J. Sel. Areas Commun. 35(7), 1459-1471 (2017)

3. F Wei et al., Virtual MIMO in multi-cell distributed antenna systems: coordinated transmissions with large-scale CSIT. IEEE J. Sel. Areas Commun. 31(10), 2067-2081 (2013)

4. D Mark, Paired Carrier Multiple Access (PCMA) for Satellite Communications (Proc. Pacific Telecommunications Conference, Honolulu, Hawaii, 1998), pp. 787-791

5. HI Jeon, JG Ryu, DG Oh, Interference cancellation scheme for commonband satellite VSAT network considering HPA nonlinear memory effects (satellite telecommunications). IEICE Tech Rep 115(241), 115-122 (2015)

6. B Nadler, Nonparametric detection of signals by information theoretic criteria: performance analysis and an improved estimator. IEEE Trans. Signal Process. 58(5), 2746-2756 (2010)

7. W Chen, KM Wong, JP Reilly, Detection of the number of signals: a predicted eigen-threshold approach. IEEE Trans. Signal Process. 39(5), 10881098 (2002)

8. L L Dong et al., Mixed Signal Detection Based on Second-Order Cyclostationary Features (Proc. IEEE Military Communications Conference (MILCOM), Baltimore, MD, 2014), pp. 682-687

9. L Dong et al., Mixed Signal Detection and Carrier Frequency Estimation Based on Spectral Coherent Features (Proc. IEEE International Conference on Military Communications, Tampa, Florida, 2015), pp. 263-268

10. H Luan et al., Source Number Estimation in Single Channel Blind Source Separation (Proc. IEEE Image and Signal Processing Conference (CISP), Yantai, China, 2010), pp. 4445-4449

11. G Fan, H Quan, H Zhang, Improved Signal Detection Scheme for Co-Channel Interference Based on Fourth-order Cyclic Cumulants (Proc. International Conference on Mechatronics Engineering and Information Technology, Dalian, China, 2017), pp. 529-533

12. $\mathrm{L} W u$, et al, Source number estimation algorithm in wavelet domain for singlechannel mixed-signal (Proc. IEEE Communication Technology Conference (ICCT), Chengdu, 2012), pp. 1048-1051

13. M Mohammadkarimi, E Karami, O Dobre, M Win, Number of transmit antennas detection using time-diversity of the fading channel. IEEE Trans. Signal Process. 65(15), 4031-4046 (2017)

14. S Miao et al., Fourth Order Cumulants in Distinguishing Single Carrier from OFDM Signals (Proc. IEEE Military Communications Conference, San Diego, CA, 2008), pp. 1-6

15. C Duan, Y Zhan, The response of a linear monostable system and its application in parameters estimation for PSK signals. Phys. Lett. A 380(14), 1358-1362 (2016)

16. JB Destro-Filho, On the blind estimation of baud-rate equalizer performance. Eurasip J. Adv. Signal Process. 12(2003), 1448-1459 (2002)

17. H Meyr, M Moeneclaey, S Fechtel, Digital communication receivers: synchronization, channel estimation, and signal processing (John Wiley \& Sons Press, New York, 1997)

18. I Nasr et al., Performance study of a near maximum likelihood code-aided timing recovery technique. IEEE Trans. Signal Process. 64(3), 799-811 (2016)

19. J Proakis, M Salehi, Digital Communications, 5th edn. (McGraw-Hill, New York, 2007)
20. S Ananthram, BM Sadler, Hierarchical digital modulation classification using cumulants. IEEE Trans. Commun. 48(3), 416-429 (2000)

21. X Xinli, J Feng, L Jiang, Automatic Digital Modulation Classification for ORS Satellite Relay Communication (Proc. IEEE International Conference on Wireless Communications \& Signal Processing, Nanjing, China, 2015), pp. 1-5

22. D Dibyajyoti, PK Bora, R Bhattacharjee, Cumulant Based Automatic Modulation Classification of QPSK, OQPSK, 8-PSK and 16-PSK (PrOc. IEEE International Conference on Communication Systems and Networks, Bangalore, 2016), pp. 1-5

23. DC Chang, PK Shih, Cumulants-based modulation classification technique in multipath fading channels. IET Commun. 9(6), 828-835 (2015)

24. M Zaerin, B Seyfe, Multiuser modulation classification based on cumulants in additive white Gaussian noise channel. IET Signal Proc. 6(6), 815-823 (2012)

25. $L$ Zheng et al., Analysis and its application of performance degradation in BPSK communication systems under monofrequency interference (in Chinese). J. Astronaut. 32(1), 136-141 (2011)

26. M Marey, OA Dobre, Blind modulation classification algorithm for single and multiple-antenna systems over frequency-selective channels. IEEE Signal Process Lett. 21(9), 1098-1102 (2014)

27. E Soltanmohammadi, M Naraghi-Pour, Blind modulation classification over fading channels using expectation-maximization. IEEE Commun. Lett. 17(9)، 1692-1695 (2013)

28. Z Guopeng et al., Optimal power control for delay-constraint machine type communications over cellular uplinks. IEEE Commun. Lett. 20(6), 1168-1171 (2016)

\section{Submit your manuscript to a SpringerOpen ${ }^{\circ}$ journal and benefit from:}

- Convenient online submission

- Rigorous peer review

- Open access: articles freely available online

- High visibility within the field

- Retaining the copyright to your article

Submit your next manuscript at $>$ springeropen.com 